\title{
A real-time assay for neutrophil chemotaxis
}

\author{
Patricia M. Cano, Amandine Vargas, and Jean-Pierre Lavoie \\ Department of Clinical Sciences, Faculty of Veterinary Medicine, Université de Montréal, Quebec, Canada
}

BioTechniques 60:245-251 (May 2016) doi 10.2144/000114416

Keywords: neutrophils; chemotaxis; xCELLigence system; real-time impedance measurement

Supplementary material for this article is available at www.BioTechniques.com/article/114416.

Neutrophils are the predominant cells during acute phases of inflammation, and it is now recognized that these leukocytes play an important role in modulation of the immune response. Directed migration of these cells to the sites of injury, known as chemotaxis, is driven by chemoattractants present at the endothelial cell surface or in the extracellular matrix (ECM). Since uncontrolled or excessive neutrophil chemotaxis is involved in pathological conditions such as atherosclerosis and severe asthma, studying the chemical cues triggering neutrophil migration is essential for understanding the biology of these cells and developing new anti-inflammatory therapies. Although several methods have been developed to evaluate neutrophil chemotaxis, these techniques are generally labor-intensive or alter the native form of these cells and their physiological response. Here we report a rapid, non-invasive, impedance-based, and label-free assay for real-time assessment of neutrophil chemotaxis.

Leukocyte migration toward infected or injured tissues is vital for host defense response and wound healing. However, leukocyte migration may also lead to pathological conditions such as cancer and chronic inflammatory diseases by triggering or prolonging tissue injury and remodeling. As first responders in acute phases of inflammation, neutrophils rapidly migrate from the blood stream to the sites of injury. Recent discoveries have revealed the ability of these cells to shape the immune response by playing an important role at the interface between innate and adaptive immunity (1). It has also been demonstrated that uncontrolled neutrophil migration may contribute to pathological conditions such as atherosclerosis, metabolic syndrome, severe asthma, and tumorigenesis (2-5).

Cell migration requires adhesive receptor-ligand interactions between leukocytes and either endothelial cells or the extracellular matrix (ECM) (6). Many chemical factors have been found to stimulate neutrophil migration. Interleukin 8 (IL-8) is one of the most potent chemoattractant in humans and several animal species including rabbits, cows, and horses $(6,7)$. Different types of migration have been defined according to whether migration occurs directionally or randomly (8). Directed migration occurs toward increasing concentrations of a soluble attractor. This form of movement is known as chemotaxis, and it is essential for leukocyte trafficking as well as the initiation and persistence of the immune response. Leukocyte migration may also be actively directed against the source of the gradient, which is known as fugetaxis. This type of movement has been suggested as a mechanism for down-regulation of inflammation (9). Random migration, referred to as chemokinesis, occurs when the attractor is present uniformly, and it is likely involved in immune surveillance. In vitro studies have shown that a chemoattractant such as Interleukin 8 (IL-8) can induce chemotaxis or chemokinesis according to the presence or absence of a gradient (10) or fugetaxis when it is present at higher concentrations (11).

Given the implication of neutrophils in several human and animal diseases, studying the signals that trigger excessive migration of these cells offers great possibilities for developing new strategies to modulate neutrophil chemotaxis. Thus, the development of technologies that provide reliable and prognosticative information regarding drug-cell interactions is key to discarding ineffective candidates early on and improving the success rate of biomedical research (12).

The Boyden chamber assay was the first reference method available for the study of cell migration. It is based on the measurement of cell transmigration from one compartment to another across a porous filter membrane in response to the presence of a chemotactic agent (13). In order to improve migration quantification and allow cell observation, other techniques, such as those using the Zigmond chamber (14), hydrogel (15), or the Dunn chamber (16), were subsequently developed. However, these methods are limited in terms of reproducibility given the difficulty in creating stable and robust gradients. To overcome these shortcomings, other approaches, such as TAXIScan (17), microfluidic-based systems (e.g., IBIDI and blood drop assays) (18-20), and 3-D collagen gels (21) have been developed in recent years. Despite improvements in gradient stability, measurement accuracy, and physiological relevance, most of these newer assays

\section{METHOD SUMMARY}

Here we present a novel method for real-time assessment of neutrophil chemotaxis. Our assay is based on the measurement of variations in electrical impedance resulting from cell adherence to the underside of a membrane as neutrophils migrate toward a chemoattractant. 
remain expensive, technically challenging, and require experienced users for experimental set-up and data analysis.

An alternative to these cell migration assays based on the measurement of electrical impedance has also emerged recently. Electric cell-substrate impedance sensing (ECIS) systems such as ECIS/ Taxis (22) and xCELLigence act by applying a harmless electrical current on gold electrodes that is impeded by the adherence of cells to the electrodes $(23,24)$. The degree of impedance reflects the number and the morphology of adhered cells (12). Thus, adherence of live cells in their native form can be monitored in real time, giving better insights into cell function kinetics. Unlike other methods, ECIS assays have multiple applications as changes in cell adhesion and morphology may be used to analyze cell proliferation, cytotoxicity, and migration (Roche Application Note. Real-time, labelfree monitoring of cellular invasion and migration with the xCELLigence system. Nat. Methods. 2002) (25-28). Compared to other ECIS systems, xCELLigence, developed by ACEA Biosciences in partnership with Roche Applied Science, allows easier and faster experimental analysis with user-friendly software without the need for lengthy preparation and stabilization steps. This system has been validated for analysis of macrophage and monocyte chemotaxis (28), but has yet to be adapted for neutrophils.

Here we present a reproducible, rapid, and easy impedance-based assay to specifically measure neutrophil chemotaxis in real time with the xCELLigence system. Unlike macrophages and monocytes, neutrophils are non-adherent cells; therefore, the direct use of the commercial material is not possible (24,25-28), and several optimization steps are required. Given the difficulty in obtaining large amounts of human neutrophils, our experiments were performed using equine neutrophils. These cells show great similarities with human and murine neutrophils, and they are used to investigate neutrophil-associated respiratory diseases in humans (29-32).

\section{Materials and methods}

\section{Ethics statement}

All experiments were performed with healthy mixed-breed mares belonging to the research herd of the Faculty of Veterinary Medicine of the University of Montreal. All animal experimental procedures followed the guidelines of the Canadian Council on Animal Care and were approved by the Animal Care Committee of the Faculty of Veterinary Medicine of the University of Montreal (Rech-1716).

\section{Neutrophil isolation}

Thirty-five milliliters of equine blood was collected from a jugular vein into heparinized sterile tubes. Peripheral neutrophils were isolated by Ficoll gradient centrifugation according to the manufacturer's instructions (Ficoll-Paque PREMIUM 1084; GE Healthcare Biosciences, Mississauga, Canada). The high-density layer containing granulocytes and erythrocytes was recovered, and erythrocytes were lysed with distilled water (Invitrogen/Thermo Fisher Scientific, Burlington Canada). Cells were then suspended in PBS (Life Technologies, Burlington, Canada) supplemented with 2 mM EDTA (Life Technologies) and 0.5\% BSA (Sigma-Aldrich, Oakville, Canada) before cell count and viability were measured with an ADAM Cell Counter (Montreal Biotech Inc., Montreal, Canada). Following this protocol, neutrophil purity was $>99 \%$, and viability was $>98 \%$. Finally, neutrophils were suspended at a suitable concentration in complete medium made with RPMI 1640 (Life Technologies) supplemented with 200 mM L-glutamine (Life Technologies), $100 \mathrm{U} /$ $\mathrm{mL}$ penicillin-streptomycin (Pen Strep) (Life Technologies), and 10\% low-endotoxin, heat-inactivated FBS (Life Technologies) before the chemotaxis assay.

\section{Real-time chemotaxis assay}

Chemotaxis assays were performed in cell invasion and migration (CIM)-plates (CIM-Plate 16; ACEA Biosciences Inc., San Diego, CA) using an xCELLigence RTCA-DP instrument (ACEA Biosciences Inc.). A CIM-plate comprises two separate parts: an upper chamber with a microporous membrane (pore size $=8 \mu \mathrm{m}$ ) embedded with gold microelectrodes on its underside and a lower chamber. The plate is assembled by locking the upper chamber on top of the lower chamber. This migratory assay is based on a small and harmless electrical current applied between the electrodes. Cell adherence to this surface while migrating from the upper chamber toward a chosen chemoattractant placed

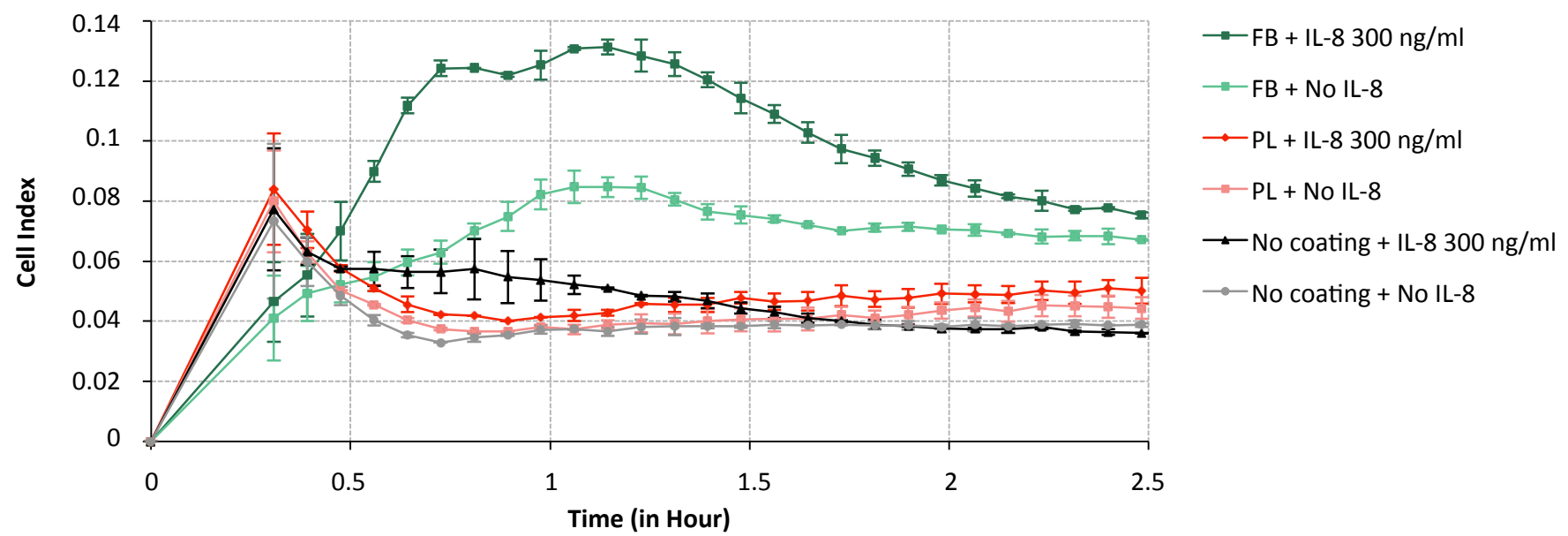

Figure 1. Effect of different coating agents on neutrophil adherence and detection. Prior to neutrophil addition to CIM-Plate 16 plates, the lower surface of the membrane of the upper chamber was coated with $50 \mu \mathrm{g} / \mathrm{mL}$ fibronectin (FB) (light and dark green lines) or $0.01 \%$ poly-L-lysine $(\mathrm{PL})$ (light and dark red lines). The assay was then performed using $2 \times 10^{5}$ cells in the upper wells and in the presence of $300 \mathrm{ng} / \mathrm{mL}$ interleukin 8 (IL-8) in the lower wells (dark green and dark red lines) or in the absence of IL-8 (light green and light red lines). Two negative controls, one without both coatings but with IL-8 (in black) and another without coating and without IL-8 (in gray) were also added to the experiment. The cell index $(\mathrm{Cl})$ was measured as cell electrical impedance every 5 min over $2.5 \mathrm{~h}$. The results are shown as the mean $\mathrm{Cl}$ for two technical replicates \pm SD. 


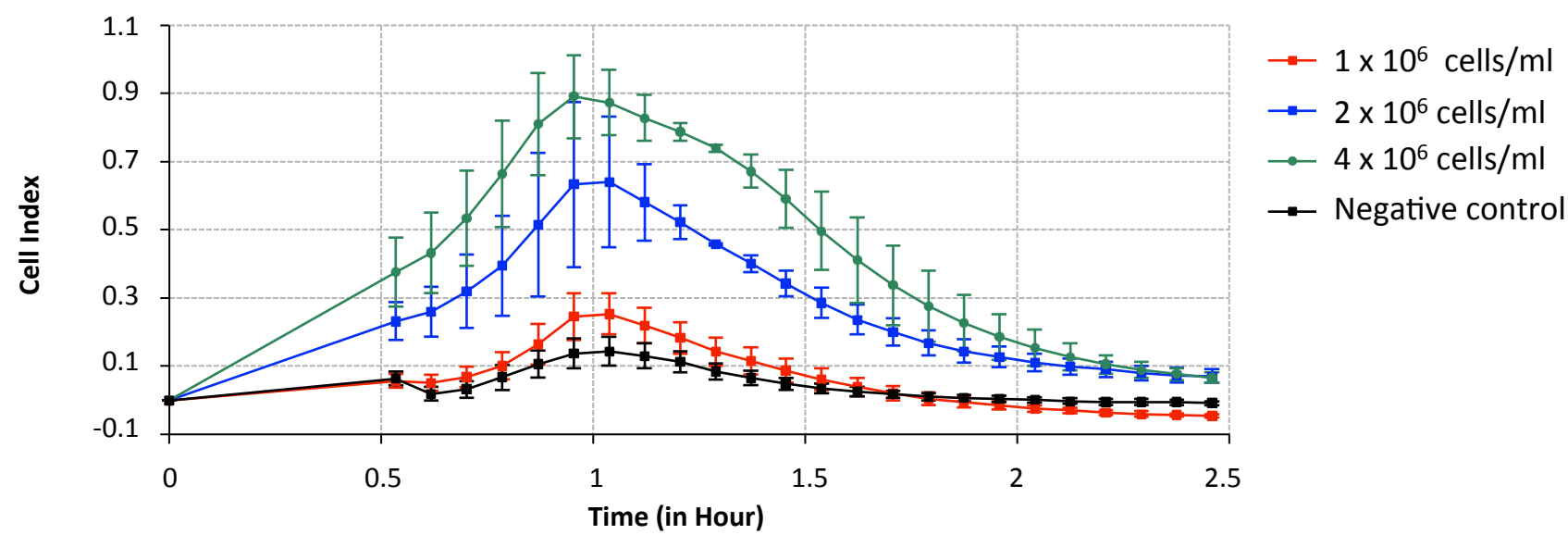

B

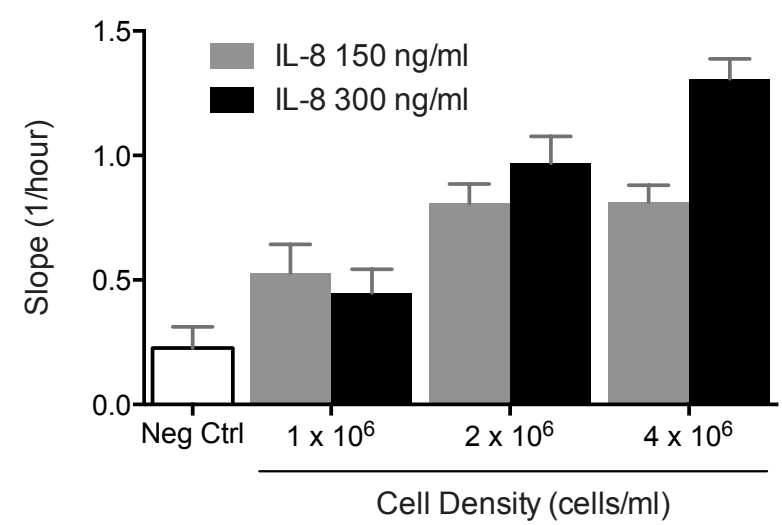

C

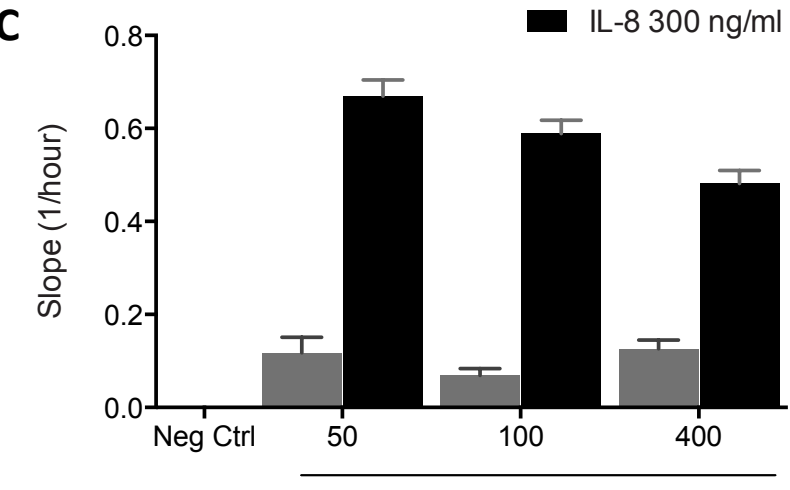

Fibronectin $(\mu \mathrm{g} / \mathrm{ml})$

Figure 2. Assay optimization: coating and cell density. (A) Cell density was optimized, with increasing neutrophil densities in the upper wells and increasing concentrations of interleukin 8 (IL-8) in the lower wells. The membrane was coated with $50 \mu \mathrm{g} / \mathrm{mL}$ fibronectin. A negative control with $4 \times 10^{6}$ cells/ $\mathrm{mL}$ and without IL-8 was included to this experiment (Neg Ctrl). The cell index (Cl) was measured as cell electrical impedance every $5 \mathrm{~min}$ for $2.5 \mathrm{~h}$. The results are shown as the mean of two technical replicates \pm SD. For graphical clarity, only the results obtained with $300 \mathrm{ng} / \mathrm{mL} I \mathrm{~L}-8 \mathrm{are}$ depicted here. (B) Slope analysis was performed using the RTCA software. The results obtained with both $150 \mathrm{ng} / \mathrm{mL}$ and $300 \mathrm{ng} / \mathrm{mL}$ IL-8 were analyzed. (C) Three concentrations of fibronectin were tested for coating the lower surface of the upper chamber membrane. Neutrophils $\left(4 \times 10^{6} \mathrm{cells} / \mathrm{mL}\right) \mathrm{were}$ added to the upper wells, and increasing concentrations of IL-8 were added to the lower wells. A negative control with $50 \mu \mathrm{g} / \mathrm{mL}$ fibronectin but without IL-8 was added to the experiment (Neg Ctrl). Slope analysis was performed using the RTCA software. The results are shown as mean of two technical replicates \pm SD.

in the lower chamber is measured. The more cells that accumulate on the underside of the membrane, the more the electrical current is impeded by the insulating properties of cellular plasma membranes. The overall cell-induced impedance is measured in real time as the cell index (Cl), a dimensionless parameter. More details concerning impedance measurements with the xCELLigence system can be found in Xi et al. (12).

As neutrophils are non-adherent cells, the underside of the membrane was coated with different coating agents prior to each experiment, as described below.

Equine IL-8 recombinant protein (Thermo Fisher Scientific, Rockford, IL) and leukotriene B4 (LTB4) (EMD Millipore, Etobicoke, Canada) were diluted to concentrations ranging from $50 \mathrm{ng} / \mathrm{mL}$ to $450 \mathrm{ng} /$ $\mathrm{mL}$ and from $1 \times 10^{-8} \mathrm{M}$ to $1 \times 10^{-6} \mathrm{M}$, respectively, in filtered $1 \times$ PBS with $10 \%$ low-endotoxin, heat-inactivated FBS. Then,
$160 \mu$ l diluted IL-8 or LTB4 was added to the lower chambers in duplicate. Following upper chamber attachment, background signal was measured by adding $35 \mu \mathrm{l}$ pre-warmed RPMI medium supplemented with L-glutamine, Pen Strep, and 10\% low-endotoxin, heat-inactivated FBS. Then, $100 \mu$ neutrophil suspension was placed in the upper chambers. Unless stated otherwise, cell density was optimized to $1.2 \times 10^{6}$ cells per well, which corresponds to a final concentration of $4 \times 10^{6} \mathrm{cell} / \mathrm{s} / \mathrm{mL}$. When testing chemokinesis, IL-8 was added to both the upper and lower chambers, whereas IL-8 was only added to the upper chambers when testing fugetaxis. The $\mathrm{Cl}$ was measured with an $\mathrm{xCELLigence}$ RTCA-DP instrument. Frequency and duration of data collection are indicated for each experiment. A more detailed version of the working protocol may be found in the Supplementary Material.
After data acquisition, slope analysis to identify the point where the maximal $\mathrm{Cl}$ value was reached was performed using the $x$ CELLigence RTCA software (ACEA Biosciences).

\section{Membrane coating}

Fibronectin (Sigma-Aldrich) and poly-Llysine (Sigma-Aldrich) were tested as coating agents for the underside of the CIM-plate membrane, as these agents have been reported to induce adhesion of neutrophils (33-35). Coating agents were diluted to the desired concentrations in sterile 1× PBS: fibronectin to $50 \mu \mathrm{g} / \mathrm{mL}$ and poly-L-lysine to $0.01 \%$. Fifty microliters of the diluted fibronectin solution was used to coat each well on the underside of the membrane; plates were incubated overnight at $4^{\circ} \mathrm{C}$ and then washed 3 times with cold sterile PBS just before the assay. Similarly, $50 \mu$ l of the diluted poly-Llysine solution was also used to coat the 
membrane, but plates were then incubated 5 min at room temperature. The membranes were then washed 3 times with cold PBS, and plates were kept in PBS overnight at $4^{\circ} \mathrm{C}$ before the assay. As fibronectin gave the best results, increasing concentrations of this coating agent ranging from $50 \mu \mathrm{g} / \mathrm{mL}$ to $400 \mu \mathrm{g} / \mathrm{mL}$ were also evaluated.

\section{Results and discussion}

Assay optimization

The aim of this work was to optimize an assay to assess chemotactic movements of neutrophils. Several modifications of previously reported protocols were required to assess neutrophil chemotaxis. First, coating of the underside of the CIM-plate membranes was optimized to detect neutrophil adherence resulting from chemotactic migration. Without coating, $\mathrm{Cl}$ values remained low (Figure 1, gray and black lines), thus confirming a low natural adherence of neutrophils to the electrodes. In extravascular tissues, leukocytes migrate along chemotactic gradients by binding to proteins in the ECM such as fibronectin, which has been used as a coating agent in several neutrophil migration assays and imaging protocols (33-35). Poly-L-lysine, a synthetic amino acid polymer, has also been used as an attachment factor to study neutrophil adhesion $(33,34)$. In our experiments, poly-L-lysine coating (Figure 1, red line) did not allow the detection of neutrophil migration. In contrast, fibronectin coating enabled the detection of neutrophil migration in response to IL-8 (Figure 1, dark green line). These results are in agreement with previous studies that reported better suitability of fibronectin for adhesive interactions with neutrophils than other ECM proteins (reviewed by Lindbom and Werr in Reference 36). There was some cell adherence that could be measured with fibronectin in the absence of IL-8 (Figure 1, light green line). Preliminary results using microscopy show neutrophils passively cross the membrane in the absence of IL-8 and cells sediment in the lower chamber after $15 \mathrm{~min}$. Sedimentation is not detected by the $x$ CELLigence system since only activated cells express surface receptors that allow their adherence to be detected; therefore, this IL-8 independent signal might reflect basal activation levels of neutrophils possibly caused by the isolation procedure or the presence of FBS in the media. These background levels did not interfere with $\mathrm{Cl}$ measurement in the presence of IL-8 (Figure 1, dark green line), especially when the signal was further optimized (Figures 2, 3, and 4). However, while detecting neutrophil chemotaxis, $\mathrm{Cl}$ values were below the lower value recommended by the manufacturer (0.2), likely resulting from insufficient cell density or an inappropriate fibronectin concentration. Increasing cell density from $6 \times 10^{5}$ cells $/ \mathrm{mL}$ to $1 \times 10^{6}$ cells $/ \mathrm{mL}$ was sufficient to obtain $\mathrm{Cl}$ values above 0.2 (Figure 2A). Slope analysis, area under the curve, and maximal $\mathrm{Cl}$ values were compared for data interpretation. Although all methods led to similar conclusions (data not shown), we selected slope analysis for follow-up experiments as it reflects not only maximal $\mathrm{Cl}$ values, but also migratory speed. Slope analysis was performed on $\mathrm{Cl}$ curves at points where the maximum value was reached. Iqbal and colleagues previously validated this analysis method when they examined chemotaxis of different leukocyte populations with the xCELLigence instrument and a scanning electron microscope at different time points (28). Optimal results in terms of maximal slope values and sensitivity to increasing

\section{ISOTYPE SWITCHING}

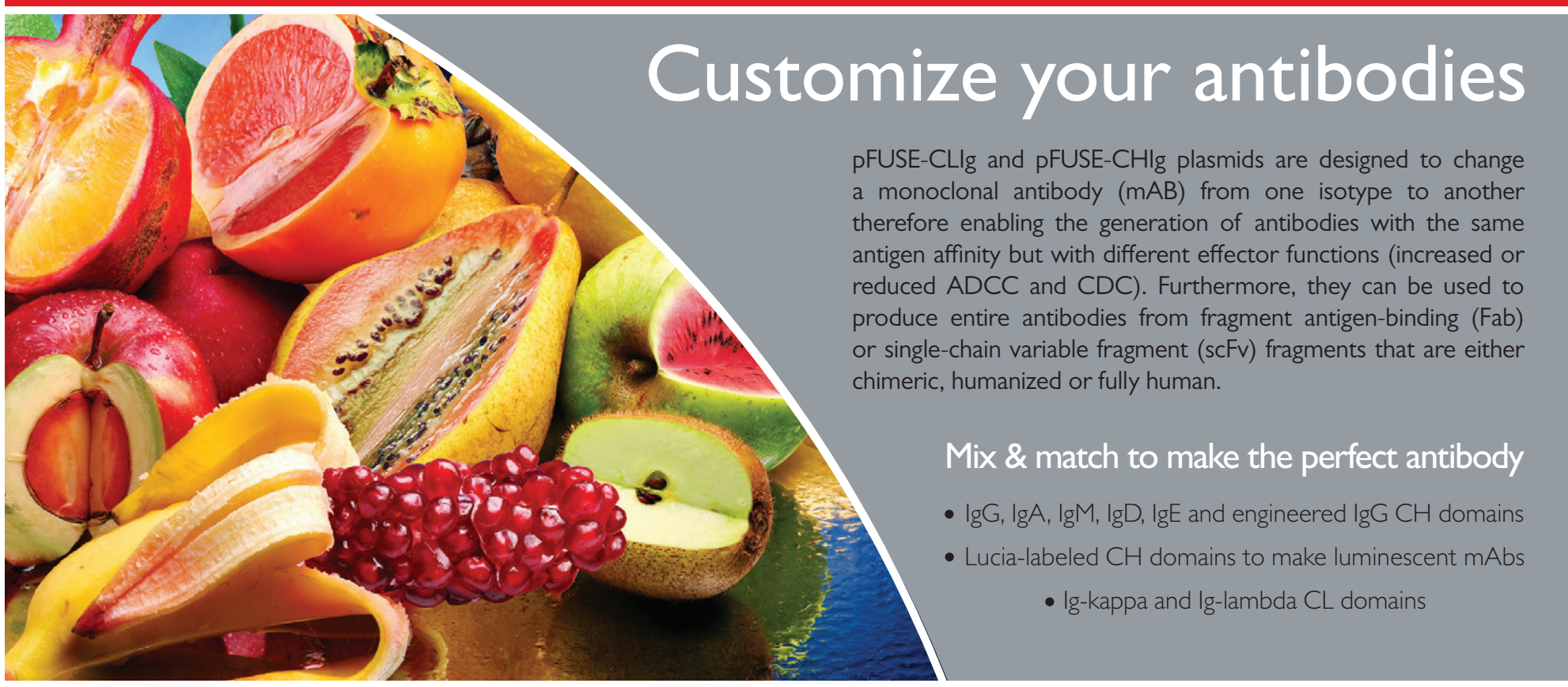


A

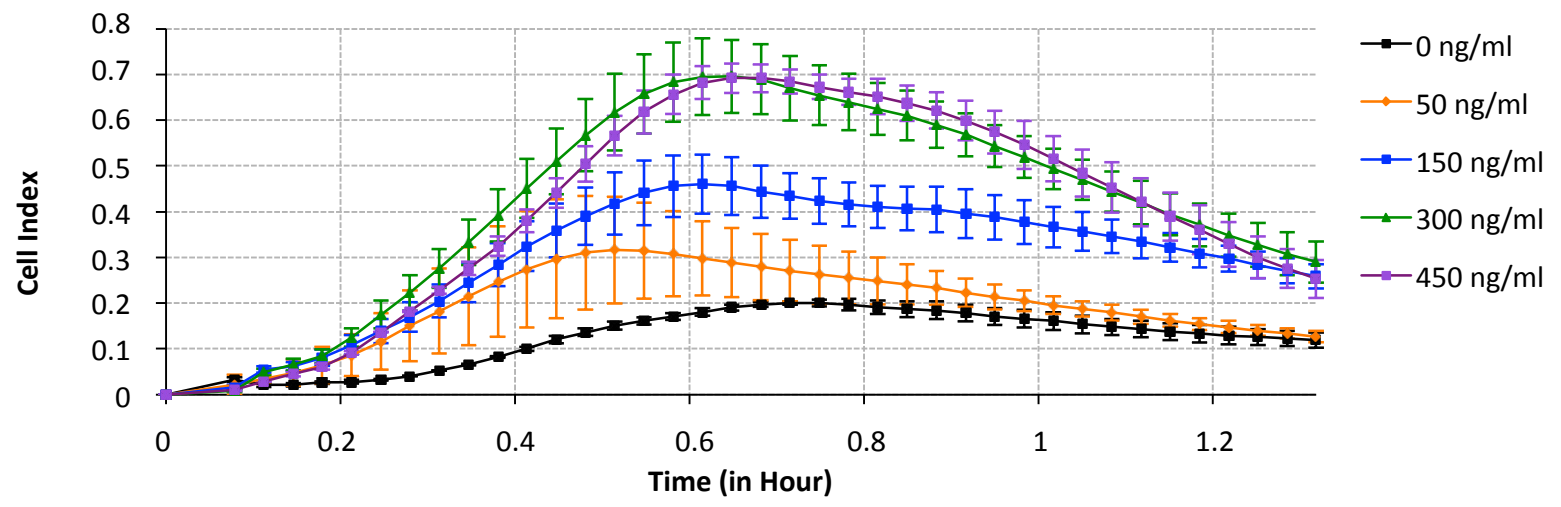

B

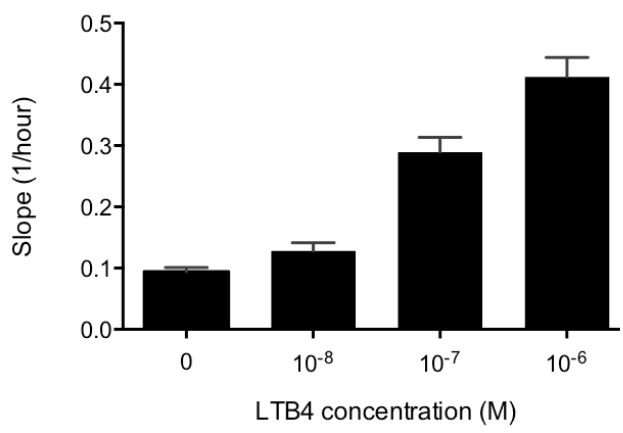

C

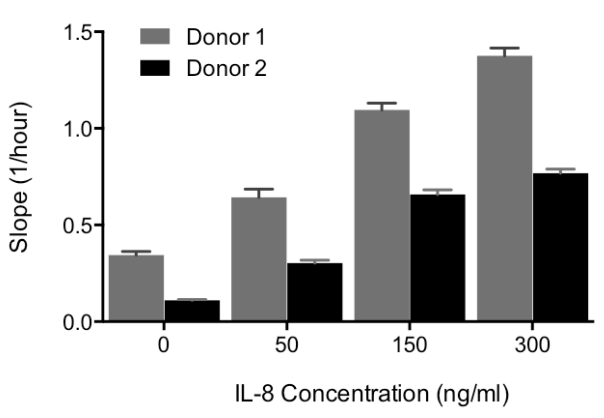

D

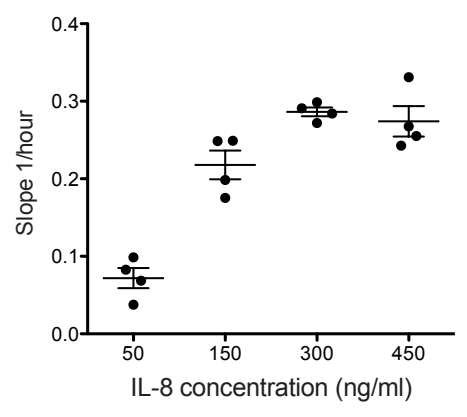

Figure 3. Dose-dependent chemotactic effect of interleukin 8 (IL-8) and leukotriene B4 (LTB4) on neutrophils. The membrane was coated with fibronectin $(50 \mathrm{\mu g} / \mathrm{mL})$, neutrophils $\left(4 \times 10^{6}\right.$ cells $\left./ \mathrm{mL}\right)$ were added to the upper wells, and increasing concentrations of IL-8 (A) or LTB4 (B) were placed in the lower wells. The cell index $(\mathrm{Cl})$ was measured as cell electrical impedance every 2 min for $1 \mathrm{~h}, 20$ min. (C) Slope analysis was performed with RTCA software for two independent experiments with IL-8. The results are shown as the mean of two technical replicates \pm SD. (D) A reproducibility assay was performed using four technical replicates of the same assay at each of four IL-8 concentrations. The results are shown as the mean \pm SD.

concentrations of IL-8 were obtained with 4 $\times 10^{6} \mathrm{cells} / \mathrm{mL}$ (Figure 2B). This cell density is similar to that used in other migration assays with human neutrophils $\left(6 \times 10^{6}\right.$ cells $/ \mathrm{mL}$ ) (21) and equine neutrophils (3 $\left.\times 10^{6} \mathrm{cells} / \mathrm{mL}, 2 \times 10^{6} \mathrm{cells} / \mathrm{mL}\right)(31,37)$, and in other impedance-based assays with monocytes and macrophages $\left(8 \times 10^{6}\right.$ cells) $\mathrm{mL}$ ) (28). Interestingly, increasing concentrations of fibronectin were not associated with higher slope values (i.e., increased neutrophil chemotaxis) (Figure 2C). Fifty micrograms per milliliter fibronectin allowed maximal detection of neutrophil migration and also the best sensitivity to increasing concentrations of IL-8. Coating the upper side of the membrane did not allow the detection of neutrophil chemotaxis as no signal could be observed when coating was performed on both sides of the membrane or on the upper side only (data not shown). These experiments highlight the specificity of the assay in measuring only adherence of the cells to the underside of the membrane resulting from chemotactic migration. Although optimized for equine neutrophils, our method should also be suitable for murine and human cells given the high functional homology among mammalian neutrophils, especially regarding the insulating properties of cellular plasma membranes (29). However, it is important to note that minor adjustments in terms of chemoattractant concentration, migration time, and coating agent may be necessary. If magnetic beads are used for neutrophil isolation, the use of a negative control is essential as this technique may induce minor cellular activation prior to the chemotaxis assay.

\section{Dose-dependent chemotactic effect of IL-8 and LTB4}

To test the sensitivity of our assay, neutrophil chemotaxis was measured in response to increasing concentrations of IL-8 and LTB4 (Figure 3). A dose-dependent response with concentrations ranging from $0 \mathrm{ng} / \mathrm{mL}$ to $450 \mathrm{ng} / \mathrm{mL}$ was observed in the presence of IL-8 (Figure 3A). At 450 $\mathrm{ng} / \mathrm{mL}$, no further increase in neutrophil migration was detected, suggesting that chemotactic properties of neutrophils may have been saturated at concentrations $>300$ ng $/ \mathrm{mL}$. Another important chemoattractant of neutrophils is the lipid mediator
LTB4 (38). When tested with the xCELLigence instrument, LTB4 induced neutrophil chemotaxis in a dose-dependent manner with concentrations raging from $1 \times 10^{-8}$ $\mathrm{M}$ to $1 \times 10^{-6} \mathrm{M}$ (Figure $3 \mathrm{~B}$ ).

The reproducibility of our assay was ascertained using four replicates of the same assay (Figure 3D), with slopes being similar in replicates for each condition. When comparing the dosedependent chemotactic effect of IL-8 between two independent donors, differences in maximal $\mathrm{Cl}$ value and migratory speed were observed, as depicted by slope differences (Figure 3C). These variations most likely reflect individual differences in basal activation of neutrophils and biological specificities between donors. Similar observations have been reported with human monocytes when using the xCELLigence instrument (28). These results emphasize the importance of real-time assays, without which early or delayed signals could be missed. Also, compared with other leukocytes such as macrophages (28), neutrophil chemotaxis occurred rapidly, highlighting the importance of these cells as a first line 


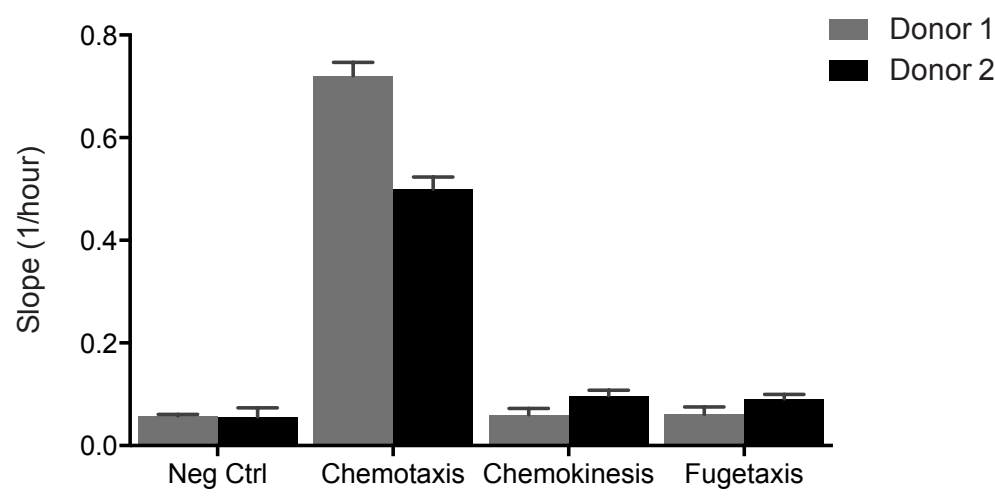

\begin{tabular}{c|ccccc}
$\mathrm{IL}-8$ & Upperwell & - & - & + & + \\
$300 \mathrm{ng} / \mathrm{ml}$ & Lowerwell & - & + & + & -
\end{tabular}

Figure 4. Detection of other cellular migratory movements. The membrane was coated with fibronectin $(50 \mu \mathrm{g} / \mathrm{mL})$ and neutrophils $\left(4 \times 10^{6} \mathrm{cells} / \mathrm{mL}\right)$ were added to the upper wells as described previously. In this experiment, $300 \mathrm{ng} / \mathrm{mL}$ interleukin 8 (IL-8) was added only to the lower wells in order to measure chemotaxis, in both the upper and lower wells to measure chemokinesis, or only to the upper wells to measure fugetaxis. No IL-8 was added in the control condition ( $\mathrm{Neg} \mathrm{Ctrl}$ ). The cell index $(\mathrm{Cl})$ was measured as cell electrical impedance every 2 min for $2.5 \mathrm{~h}$. Slope analysis was performed with RTCA software for two independent experiments. The results are shown as the mean of two technical replicates \pm SD.

of defense. Given the speed at which neutrophil chemotaxis takes place, the 30 min incubation time recommended by the manufacturer for cell sedimentation prior to each experiment for other cell types was removed from our protocol. Otherwise, chemotactic movements were already achieved, and the increase in neutrophil migration in the presence of IL-8 could not be detected.
Assay chemotaxis specificity

In addition to chemotaxis, neutrophils also have the ability to migrate randomly (chemokinesis) or to migrate away from a chemotactic agent (fugetaxis), depending on the concentration of the chemoattractant and the intensity of the gradient $(10,11)$. Therefore, the specificity of our assay for measurement of chemotaxis was evaluated with a concentration of IL-8 known to induce only this type of movement in equine neutrophils (37). Detection of artifactual signals was tested with IL-8 placement (i) only in the lower wells, thus simulating chemotaxis, as a positive control; (ii) at similar concentrations in the upper and the lower wells for chemokinesis; and (iii) only in the upper wells to test fugetaxis. As shown in Figure 4 for two different donors, only chemotactic movements could be detected, thus confirming the specificity of the assay for measurement of neutrophil chemotaxis. However, the present protocol is not limited to the study of chemotaxis and could be adapted to neutrophil chemokinesis and fugetaxis by using lower concentrations of IL-8 for chemokinesis (10) and higher concentration for fugetaxis (11).

\section{Improve Your PCR Workflow}

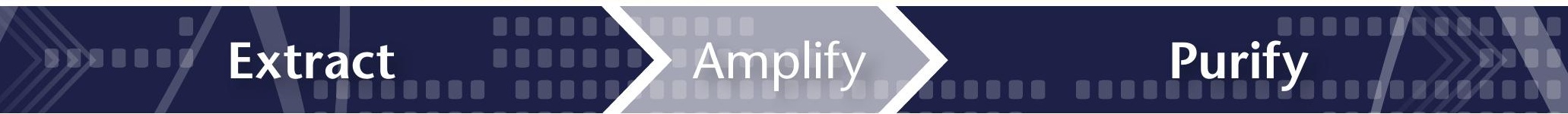

DNA Rapid Extract ${ }^{\text {"W Kit }}$

FAST • SIMPLE • EFFICIENT

\section{Diffinity DNA RapidExtract ${ }^{\mathrm{TM}}$ Kit}

Our DNA RapidExtract ${ }^{\mathrm{TM}}$ Kit lyses DNA in a single incubation step and yields PCR-ready DNA in 10 minutes.

ONE KIT for multiple sample types

- Plant (leaf, seeds, pulp) - Hair Follicle - Bird Feather

- Blood (EDTA or heparin) - Animal Tissue • Feces

- Buccal Swab

Easy-to-use protocol for multiple sample types

No special storage is required

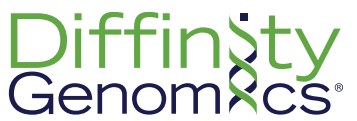

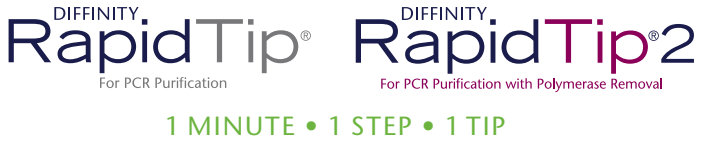

\section{Diffinity RapidTip ${ }^{\circledR}$ and RapidTip ${ }^{\circledR}$}

Diffinity Genomics ${ }^{\circledR}$ RapidTips remove dNTPs, primers, primer-dimers and DNA polymerase from your PCR sample while providing highrecovery of desired DNA fragments for downstream applications (genotyping, T-A cloning, and Sanger cycle sequencing reactions).

No bind-wash-elute, enzymes, or magnetic beads

Each tip is pre-packed with Differentially Functionalized Nano-Porous Particles for use right out of the box

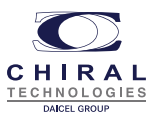
trademarks of Chiral Technologies, Inc. 
Here we have described an impedancebased protocol to monitor the chemotaxis of label-free neutrophils in real time. Unlike previous methods, our assay has the advantage of being reproducible, fast, user friendly, and easy to analyze and interpret. This technique could facilitate the selection of drug candidates for regulating the influx of neutrophils, aiding the development of therapies against chronic inflammatory diseases. Our results also suggest that this type of assay could be used to study other migratory movements of neutrophils, as well as migration of other non-adherent cells such as lymphocytes.

\section{Author contributions}

P.M.C. contributed to the execution and analysis of the data as well as in the writing and editing of the manuscript. A.V. was involved in the conception and execution of the experiments as well as in the writing and editing of the manuscript. J.P.L. supervised the conception, execution, and analysis of the experiments as well as the writing and editing of the manuscript.

\section{Acknowledgments}

This work was supported by the Natural Sciences and Engineering Research Council of Canada (RGPIN-2014-06-198). The authors would like to thank Melissa Brierley, Country Manager at ACEA Biosciences, Inc. for technical assistance with the $x$ CELLigence RTCA-DP instrument.

\section{Competing interests}

The authors declare no competing interests. However, ACEA Biosciences provided the CIM-plates required for the study but had no further involvement in the experimental design or data analysis.

\section{References}

1. Mócsai, A. 2013. Diverse novel functions of neutrophils in immunity, inflammation, and beyond. J. Exp. Med. 210:1283-1299.

2. Monteseirín, J. 2009. Neutrophils and asthma. J. Investig. Allergol. Clin. Immunol. 19:340-354.

3. Drechsler, M., R.T. Megens, M. van Zandvoort, C. Weber, and O. Soehnlein. 2010. Hyperlipidemia-triggered neutrophilia promotes early atherosclerosis. Circulation 122:1837-1845.

4. Gregory, A.D. and A. McGarry Houghton. 2011. Tumor-associated neutrophils: new targets for cancer therapy. Cancer Res. 71:2411-2416.

5. Talukdar, S., Y. Oh da, G. Bandyopadhyay, D. Li, J. Xu, J. McNelis, M. Lu, P. Li, et al. 2012. Neutrophils mediate insulin resistance in mice fed a high-fat diet through secreted elastase. Nat. Med. 18:1407-1412

6. Wagner, J.G. and R.A. Roth. 2000. Neutrophil migration mechanisms, with an emphasis on the pulmonary vasculature. Pharmacol. Rev. 52:349-374.

7. Brooks, A.C., K.J. Rickards, and F.M. Cunningham. 2011. CXCL8 attenuates chemoattractant-induced equine neutrophil migration. Vet. Immunol. Immunopathol. 139:141-147.

8. Petrie, R.J., A.D. Doyle, and K.M. Yamada. 2009. Random versus directionally persistent cell migration. Nat. Rev. Mol. Cell Biol. 10:538-549.

9. Vianello, F., I.T. Olszak, and M.C. Poznansky. 2005. Fugetaxis: active movement of leukocytes away from a chemokinetic agent. J Mol Med (Berl). 83:752-763.

10. Knall, C., G.S. Worthen, and G.L. Johnson. 1997. Interleukin 8-stimulated phosphatidylinositol-3-kinase activity regulates the migration of human neutrophils independent of extracellular signal-regulated kinase and p38 mitogen-activated protein kinases. Proc. Natl. Acad. Sci. USA 94:3052-3057.

11. Tharp, W.G., R. Yadav, D. Irimia, A. Upadhyaya, A. Samadani, O. Hurtado, S.Y. Liu, S. Munisamy, et al. 2006. Neutrophil chemorepulsion in defined interleukin-8 gradients in vitro and in vivo. J. Leukoc. Biol. 79:539-554

12. Xi, B., N. Yu, X. Wang, X. Xu, and Y.A. Abassi. 2008. The application of cell-based label-free technology in drug discovery. Biotechnol. J. 3:484-495.

13. Boyden, S. 1962. The chemotactic effect of mixtures of antibody and antigen on polymorphonuclear leucocytes. J. Exp. Med. 115:453-466.

14. Zigmond, S.H. 1977. Ability of polymorphonuclear leukocytes to orient in gradients of chemotactic factors. J. Cell Biol. 75:606-616.

15. Nelson, R.D., P.G. Quie, and R.L. Simmons. 1975. Chemotaxis under agarose: a new and simple method for measuring chemotaxis and spontaneous migration of human polymorphonuclear leukocytes and monocytes. J. Immunol. 115:1650-1656.

16. Zicha, D., G.A. Dunn, and A.F. Brown. 1991. A new direct-viewing chemotaxis chamber. J. Cell Sci. 99:769-775.

17. Nitta, N., T. Tsuchiya, A. Yamauchi, T. Tamatani, and S. Kanegasaki. 2007. Quantitative analysis of eosinophil chemotaxis tracked using a novel optical device - TAXIScan. J. Immunol. Methods 320:155163.

18. Agrawal, N., M. Toner, and D. Irimia. 2008. Neutrophil migration assay from a drop of blood. Lab Chip 8:2054-2061.

19. Jowhar, D., G. Wright, P.C. Samson, J.P. Wikswo, and C. Janetopoulos. 2010. Open access microfluidic device for the study of cell migration during chemotaxis. Integr Biol (Camb). 2:648-658.

20. Sackmann, E.K., E. Berthier, E.W. Young, M.A. Shelef, S.A. Wernimont, A. Huttenlocher, and D.J. Beebe. 2012. Microfluidic kit-on-a-lid: a versatile platform for neutrophil chemotaxis assays. Blood 120:e45-e53.

21. Martin, K.J.S., M.J. Muessel, C.E. Pullar, G.B. Willars, and A.J. Wardlaw. 2015. The Role of phosphoinositide 3-kinases in neutrophil migration in 3D collagen gels. PLoS ONE 10:e0116250.

22. Pietrosimone, K.M., X. Yin, D.A. Knecht, and M.A. Lynes. 2012. Measurement of Cellular Chemotaxis with ECIS/Taxis. J. Vis. Exp. pii:3840.

23. Giaever, I. and C.R. Keese. 1984. Monitoring fibroblast behavior in tissue culture with an applied electric field. Proc. Natl. Acad. Sci. USA 81:3761-3764.

24. Giaever, I. and C.R. Keese. 1993. A morphological biosensor for mammalian cells. Nature 366:591-592.
25. Atienzar, F.A., K. Tilmant, H.H. Gerets, G. Toussaint, S. Speeckaert, E. Hanon, O. Depelchin, and S. Dhalluin. 2011. The use of real-time cell analyzer technology in drug discovery: defining optimal cell culture conditions and assay reproducibility with different adherent cellular models. J. Biomol. Screen. 16:575-587.

26. Limame, R., A. Wouters, B. Pauwels, E. Fransen, M. Peeters, F. Lardon, O. De Wever, and P. Pauwels. 2012. Comparative analysis of dynamic cell viability, migration and invasion assessments by novel real-time technology and classic endpoint assays. PLoS ONE 7:e46536.

27. Atienzar, F.A., H. Gerets, K. Tilmant, G. Toussaint, and S. Dhalluin. 2013. Evaluation of impedancebased label-free technology as a tool for pharmacology and toxicology investigations. Biosensors 3:132-156.

28. Iqbal, A.J., D. Regan-Komito, I. Christou, G.E. White, E. McNeill, A. Kenyon, L. Taylor, T.S. Kapellos, et al. 2013. A real time chemotaxis assay unveils unique migratory profiles amongst different primary murine macrophages. PLoS ONE 8:e58744.

29. Styrt, B. 1989. Species variation in neutrophil biochemistry and function. J. Leukoc. Biol. 46:63-74.

30. Hirsch, G., A. Lavoie-Lamoureux, G. Beauchamp, and J.-P. Lavoie. 2012. Neutrophils Are Not Less Sensitive Than Other Blood Leukocytes to the Genomic Effects of Glucocorticoids. PLOS ONE 7:e44606.

31. Bullone, M., K. Moran, A. Lavoie-Lamoureux, and J.P. Lavoie. 2013. PI3K and MAPKs regulate neutrophil migration toward the airways in heaves. J. Vet. Intern. Med. 27:164-170.

32. Brazil, T.J., P.M. Dixon, C. Haslett, J. Murray, and B.C. McGorum. 2014. Constitutive apoptosis in equine peripheral blood neutrophils in vitro. Vet. J. 202:536-542.

33. Reumaux, D., T.W. Kuijpers, P.L. Hordijk, P. Duthilleul, and D. Roos. 2003. Involvement of FCy receptors and $\beta(2)$ integrins in neutrophil activation by anti-proteinase-3 or anti-myeloperoxidase antibodies. Clin. Exp. Immunol. 134:344-350.

34. Combaluzier, B. and J. Pieters. 2009. Chemotaxis and phagocytosis in neutrophils is independent of Coronin 1. J. Immunol. 182:2745-2752.

35. Lim, S.Y., M.J. Raftery, J. Goyette, and C.L. Geczy. 2010. S-glutathionylation regulates inflammatory activities of S100A9. J. Biol. Chem. 285:14377-14388.

36. Lindbom, L. and J. Werr. 2002. Integrin-dependent neutrophil migration in extravascular tissue. Semin. Immunol. 14:115-121.

37. Côté, O., M.E. Clark, L. Viel, G. Labbé, S.Y.K. Seah, M.A. Khan, D.N. Douda, N. Palaniyar, and D. Bienzle. 2014. Secretoglobin 1A1 and 1A1A differentially regulate neutrophil reactive oxygen species production, phagocytosis and extracellular trap formation. PLoS ONE 9:e96217.

38. Foster, A.P., P. Lees, and F.M. Cunningham. 1992. Platelet activating factor is a mediator of equine neutrophil and eosinophil migration in vitro. Res. Vet. Sci. 53:223-229.

Received 28 June 2015; accepted 05 February 2016.

Address correspondence to Jean-Pierre Lavoie, Department of Clinical Sciences, Faculty of Veterinary Medicine, Université de Montréal, CP 5000 SaintHyacinthe, Quebec, J2S 7C6, Canada. E-mail: jeanpierre.lavoie@umontreal.ca

To purchase reprints of this article, contact: biotechniques@fosterprinting.com 